\title{
LA VECCHIAIA FEMMINILE NELla POESIA TOSCANA DEL XV SECOLO
}

Recentemente il motivo della vecchiaia femminile è stato oggetto di vari studi di impronta femminista. Partendo da alcuni contributi apparsi su Memoria, dedicati al problema dell'invecchiamento femminile dal punto di vista sociologico, culturale e letterario, Ornella Marotti rileva l'esistenza di un preconcetto di sesso/genere nell'atteggiamento socio-culturale verso la vecchiaia. Da qui, secondo la studiosa, la necessità di fornire modelli positivi di vecchiaia della donna, almeno nella letteratura contemporanea soprattutto femminile. ${ }^{1}$

11 preconcetto nei confronti della vecchiaia femminile rilevato da Marotti è ampiamente attestato nella nostra tradizione letteraria in cui, la voce autoriale prevalentemente maschile, non dà spazio a rappresentazioni positive della donna anziana. Il pregiudizio letterario verso le vecchie, retaggio della tradizione classica. è assai diffuso e radicato fin dai primi secoli. Questo saggio, individuando in breve le fonti latine e la tradizione realistica medievale che hanno forgiato l'immagine negativa della vecchiaia femminile nel corso dei primi secoli, intende concentrarsi in particolare sulla poesia comica toscana del XV secolo. ${ }^{2}$ Oltre a riprendere motivi e temi della tradizione latina e medievale, i poeti toscani del Quattrocento introducono caratteri specifici e originali che riflettono il clima storico-sociale dell'epoca, arrichiscono il topos della vecchiaia femminile e anticipano motivi presenti nella letteratura del XVI secolo.

Come osservò già Vittorio Cian la satira contro le vecchie ha una storia secolare varia e curiosa riconducibile ad una duplice origine: l'antichità classica e la letteratura popolare che spesso si intersecano (310).

Nella tradizione latina troviamo numerosi attacchi alle vecchie nella poesia di Orazio: nel carme 13 del libro IV il poeta gioisce per linvecchiamento della prostituta lasciva Cinara di cui descrive i denti opachi, il corpo rugoso e i capelli bianchi. Nell'Epodo 8 il tono si fa ancora più crudo e i dettagli del corpo invecchiato più disgustosi: Canidia, la vecchia libidinosa, è carica di anni, piena di rughe e canuta, ma non sembra aver perduto il suo 
desiderio erotico; l'attività di stregoneria della vecchia laida sembra confermata dalla comparsa di Canidia in un altro epodo (5) che elenca le sue arti magiche. Anche nell'epodo 12 compare una vecchia lasciva e disgustosa dal caratteristico odore ributtante simile a quello di una capra e di altri animali maleodoranti. Tre epigrammi di Marziale (Libro III, 32. 76 e 93) si scagliano contro la vecchia dall'età incredibilmente avanzata, quasi proverbiale, e dal cattivo odore disgustoso. Negli Amores (Libro I, 8) Oviclio inveisce contro Dipsa, una vecchia prostituta e mezzana (lena) con pochi capelli in testa, che si intende di magia ed è una sfrenata bevitrice, sempre ubriaca all'alba. Properzio nel IV libro delle Elegie impreca contro Acanthis, una vecchia prostituta semi-calva (assimilata talvolta ad un lupo) che usa arti magiche e fa da mezzana alle giovani, clando loro consigli su come attirare gli uomini prima che sia troppo tardi. Per Simone de Beauvoir il particolare accanimento contro le vecchie nella letteratura latina è il prodotto di una società come quella romana, in cui la donna è vista principalmente come oggetto erotico e lá vecchiala rende la donna un monstmm fonte di odio e disgusto, un essere che dallo stato umano diventa soprannaturale, strega e maga. (131)

La polemica contro le vecchie persiste nella letteratura mediolatina e culmina nell'Ars versificatoria di Matthieu deVendôme dove, ad esemplificare la descriptio mmlieris in negativo, troviamo il vituperio della vecchia $e$ orrenda Beroe. Questo testo è praticamente canonico per le successive descrizioni disgustose della vecchia laida, puzzolente, dagli occhi colanti e denti color ruggine. ${ }^{3}$

Nel Medioevo volgare la donna anziana è soggetto della vituperatio vetulae nella poesia comico-realistica. In diretta contrapposizione con la poesia aulica stilnovista, che esaltava la giovane donna angelicata, nel genere comico prevale l'invettiva contro la vecchia. Ricordiamo il sonetto di Rustico Filippi "Dovunque vai con teco porti il cesso,/ buggeressa vecchia puzzolente", dove l'ostilitì per la vecchia e il suo cattivo odore rinviano alla tradizione latina e alla Beroe di Vendôme. Due sonetti dubbiamente attribuiti a Cecco Angiolieri ("Tutto mi strugge l'animo una vecchia", "Mándarti poss'io "l sangue in una secchia") inveiscono contro la vecchia guardiana della donna amata ma eludono riferimenti precisi all'aspetto fisico repellente. ${ }^{-}$ Non sono solo gli autori comico-realistici a cimentarsi nel motivo della vecchia orrenda, anche gli stilnovisti lanno qualche incursione nell'area del comico, per sbeffare le vecchie. Nel sonetto "Volvol te levi vecchia rabbiosa" Guicks Guinizelli si scaglia con toni realistici contro una vecchia, forse guardiana della donna amata. La vecchia ingobbita è anche veicolo di parodia clella bellezza perfetta dell'anata stilnovista nel sonetto di Guido 
Cavalcanti ("Guata Manetto quella scrignutuzza") a cui risponde per le rime "Deh guata, Ciampol, ben questal vecchiuzza", un tempo attribuito a Cecco Angiolieri ed oggi ritenuto di Vicola Muscia. Anche la poesia popolare si rivolge con toni duri e linguaggio crudo contro le vecchie, specialmente contro le guardiane della donna amata. In questi testi la vecchia è vituperata per la sua malvagità, il suo aspetto fisico disgustoso e grottesco, per il suo cáttivo odore e per la sua derivazione quasi bestiale da animali. Franco Sacchetti dimostra un particolare accanimento contro le vecchie, sia nelle Rime (si vedano le ballate "Di diavol vecchia femmina ha natura", "Qual diavol vecchic subito vi toccá" e "Tra "I bue l'asino e le pecorelle"), sia nel poemetto in ottave La battaglia delle belle domme di Firenze in cui le donne giovani hanno la meglio nello scontro con le vecchie orrende. 5 Nel Medioevo l'invettiva contro la vecchiaia femminile diventa pertanto un luogo comune letterario che si esprime nella forma della vituperatio velulae; Marti (4-13) considera la misoginia e l'accanimento contro le vecchie non tanto un reale atteggiamento negativo contro le donne anziane, quanto un esercizio retorico sul contrario: come prescritto dalle Artes dictandi ed esemplificato nella Beroe di Vendôme, all'elogio della giovane fanciulla nello stile alto si contrappone il vituperio della vecchia laida nello stile basso e realistico. Nella tradizione comico-realistica medievale mancano i motivi latini della vecchia prostituta lasciva e/o strega, e il linguaggio osceno. La poesia popolare invece, con toni assai più violenti e linguaggio crudo, attacca la vecchia guardiana della donna amata attribuendole talvolta anche l'epiteto di strega, figura che diventerà ricorrente nelle poesie comiche del $X \mathrm{~T}^{\top}$ secolo. ${ }^{6}$

Nel XT secolo è ancora la corrente comico-realistica ad accogliere gli esempi di poesia sulla vecchiaia femminile. Persiste l'atteggiamento generalmente ostile verso le donne anziane; la vecchia, sempre malvagia, orrenda e maldicente, si rivela ora più chiaramente come strega, ruffiana e/o prostituta, categorie femminili finora quasi inedite nella nostra tradizione volgare, ma rese famose nel XVI secolo da autori come Ariosto, Niccolò Franco, Aretino.-

La maggior produzione di testi poetici contro la vecchia è presente nel XV secolo in area toscana con Burchiello (1404-1448), i post-burchielleschi come Giovan Matteo di Meglio (1427-?). Antonio Cammelli ( 1436-1502) detto il Pistoia, per culminare nelle dotte sperimentazioni del Poliziano.` Gli esempi toscani combinano i topoi ormai consolidati della tituperatio vetulae medievale con elementi nuovi, come la figura della strega o della prostituta/ruffiana. Lemergere di queste figure femminili deriva in parte dal recupero della tradizione latina, in parte da una situazione storico-sociale di par- 
ticolare ostilità contro queste donne. Il motivo della vecchia strega e della prostituta/lasciva era quasi del tutto assente nella poesia comico-realistica medievale, dove gli elementi negativi riguardavano più il carattere malvagio e il fisico repellente della vecchia. La presenza nella poesia del XV secolo di figure femminili come la strega e la prostituta, spesso l'una come sinonimo dell'altra, riflette gli esempi classici di Orazio, Ovidio e Properzio, ma corrisponde anche ad una realtà storica segnata da una crescente avversione contro le vecchie-streghe. La strega, vista con sospetto dai poeti latini, era presente già nella fantasia popolare medievale. Tuttavia solo dopo il XIV secolo, col riconoscimento da parte della Chiesa della strega come persona realmente esistente, colpevole di eresia e di rapporti col diavolo, prende maggiore consistenza l'ossessione e l'accanimento contro questa figura femminile.? Da una prospettiva storica Chiara Frugoni vede la cliffusa ostilità contro le donne credute streghe come conseguenza della predicazione di personaggi come San Bernardino da Siena. (382) ${ }^{10}$ Le biografie di streghe riferivano del loro smodato appetito sessuale e delle loro relazioni sessuali degenerate col diavolo, le streghe per definizione erano orrende e vecchie; secondo un proverbio medievale noto ancor oggi in area slava "ogni vecchia è una strega". ${ }^{11}$ Il riferimento alla strega entra nella poesia del XV secolo anche attraverso la mediazione della poesia trecentesca popolare e madrigalistica, in cui l'epiteto attribuito alla vecchia guardiana della fanciulla ámata è appunto strega, senza peraltro soffermarsi a descrivere i suoi poteri o atti malefici. ${ }^{12}$ Nella Feminist Encyclopedia of Italian Literature, la strega, insieme alla prostituta (e alla vedova, donna presumibilmente più anziana) è identificata con quel tipo di donna che resiste alle definizioni sessuali facenti capo all'istituzione del matrimonio. Come figura femminile non categorizzabile da parte maschile essa è, sia socialmente che fisiologicamente, impossibile da controllare, perché soggetta solo al desiderio incontenibile di un corpo socialmente non omologato. Tale corpo non conforme viene demistificato e associato ad un contenuto mostruoso (strega come diavolo o animale molesto), da qui l'accanimento contro la strega, sia nella realtà storica, in cui la strega reniva bruciata pubblicamente al rogo, siá nei testi letterari, in cui l'invettiva augura spesso la distruzione del corpo.

\section{Burchiello}

Nella poesia di Burchiello Antonio Lanza (Polemiche e berte. 174) individua una notevole caricá innovativa che deriva dalla rielaborazione dei temi giocosi medieváli, ma ancor più dall'esperienza della vita reale e dell'anbiente popolare fiorentino. Lá critica riconduce le invettive burchiellesche contro le 
vecchie, non tanto all'influsso della vita reale quanto alla tradizione letteraria medievale. 13 I due sonetti caudati di Burchiello contro k' vecchice ("Véchial ritrosa, perfidá e maligna" e "Ardati il fuoco vecchia puzzolente") riprendono infatti ke caratteristiche dellat tituperatio étulae illustrata dai sonetti di Rustico, Guinizelli e pseudo-Cecco. In Burchiello emerge tuttavia una novità: la figura della recchia si fonde con quella della strega incantatrice e maliosa, collegando l'aversione di Burchiello per le vecchie streghe alla temperie storico-culturale di furore contro di esse diffusa dalle prediche di Bernardino.

Vecchia ritrosa, perlida e maligna, inimica d'ogni hen, invidiosal,

e strega incantatrice e maliosa, trista, stravolta, che se' pien di tigna.

Barbutal se' più folta che gramigna, gli occhi e 'I natso ti colan senza posa, puzzati el fiato, sclentatat rabbiosa se ridi pari un diavol che digrigna. E. tanto è velenosá la tua vista che ciò che miri corrompi per paodo, [che...angel non..pua....o silmista.] Ma io mi voglio di te un colabrodo, che sempre mai tha fatto viver trista, e pagner....se m'hai fatto frodo.

E di questo mi godo, perché da te si fugge tutta gente, per lo tuo marcio conno puzzolente. (45)

La vecchia maleodorante clagli occhi e naso colanti, ha le stesse caratteristiche del ritratto di Beroe della poesia mediolatina. ${ }^{1+}$ La componente del cattivo odore rinvia al vituperio di Rustico Filippi, mentre l'aggettivo "rabbiosa" ricorda la vecchia di Guinizelli. I tratti del carattere della vecchia sono fissati nel consueto schema misogino della natura maliziosa, perficla e invidiosá. Distaccandosi dalla tradizione giocosa medievale Burchiello identifica la vecchia con la strega, di cui clemistificá il corpo sia mediante il ritratto grottesco, sia nell'augurio della distruzione corporeá ("Malo mi voglio di te un colabrodo").

Nel secondo sonetto ritorna la violenta invettiva contro la vecchia strega, che come sosteneva la Chiesa, è apertamente accusata di pratiche ereticali.

Ardati il fucoco, vecchia puzzolente, Che non ti resti mai di pensar male. 
D'eresia seminando le tue scale Poi che moneta non trai dalla gente: Cieca ti fai; Dio ti faccia dolente, Fussinti tratti gli occhi, e messi in sale; Ed io fussi di te il micidiale, Acciò che fussin le tue fiamme spente: Lupo cervier non ha il veder sottile, Come tu sottilezzi ragguardando, Né da sì piccol buco, tanto umile. Pigliar diletto forte sospirando, Per ch'aggrizzando il volticel vecchile, Col borbottar mi parti' lagrimando:

Al fuoco taccomando,

O vecchia strega, o maliziosa ghiotta,

Ladra, ruffiana, malacletta botta. ${ }^{15}$

Il disgusto del ritratto deriva dal cattivo odore, come già in Rustico, in Muscia e nelle ballate popolari. Ben evidente è il tessuto di prestiti dotti: il "volticel vecchile" grinzoso è una delle caratteristiche più ricorrenti nei ritratti delle vecchie. La vecchia borbotta e guarda con vista acuta, due atteggiamenti in cui viene rappresentata la vecchia guardiana e molesta.16 In un perfetto ordine circolare il sonetto si apre e si chiude con l'invettiva e l'augurio di morire bruciata. L'accanimento contro il corpo della strega include l'estrazione degli occhi e la distruzione al rogo. Oltre che strega la vecchia è chiamata anche ruffiana, una figura femminile contigua a quella della prostituta, che prevale nel XV secolo in poeti come Matteo di Meglio e Poliziano.

\section{Poeti post-burchielleschi}

Nella limitata produzione (una trentina di componimenti) di Giovan Matteo di Meglio, esponente della poesia giocosa toscana post-burchiellesca, spicca la presenza assai diffusa di vituperi contro la vecchia malvagia, strega e ruffiana, per cui anche Matteo utilizza il sonetto caudato. Secondo Lanza ("Aspetti e figure" 410) i vituperi di Giovan Matteo dimostrano la lezione del Burchiello e del suo maestro Angiolieri, ma vi aggiungono "un'esasperata ricerca di immagini e di spunti d'un verismo crudo e talora rivoltante." (413)

Il topos della vecchia vituperata è attestato dal sonetto caudato "Vecchia azzimata, ricarclata e vizzá".

Vecchia azzimata, richardata e vizza, nata di più albumi e più veleni che tutt'altri animal bruti terreni, 
ereticha, maligna el mare di stizza,

lupa gholpina, romat, et chagna in izza.

ipocrita, strebbiata el sanzal freni,

nel tuo inferno molt alme a morte meni:

ghuai a quel chasseghuirti il chor clirizza!

Pubblichal mamma di toro é di ierro,

discesal di progenial vile e strana,

pazza, hubbriacha, villana e bastarda;

nata di hestia, chalzata cli ferro,

putta honesta et sollecita ruffiama,

pront'al mal fare, at ben pigar e 'nfingharda.

Maliosa e bugiarda!

quel ch'ell'a fato 'l fa et fallo fare.

et dove fugge 'l topo si fa dase. (19-51)

Giovan Matteo conserva le caratteristiche canoniche del ritratto morate misogino (la vecchia criticata per l'uso del belletto) e riprende l'associazione della vecchia con lá volpe e il lupo, animali fortemente simbolici nel Mediocvo (basti pensare a Dante), ma anche trasformazioni clella strega. Alla vecchia orrenda, generatrice di esseri non umani, già presente in Rustico Filippi ("in corpo credo figlinti le volpe"), Matteo aggiunge anche la provenienza genetica da animali. Anche l'aggettivo "vizza", e la sperimentazione linguistica delle rime aspre, cleriva dalla tradizione comico-realistica trecentesca (ricordiamo la "scrignutuzza" di Cavalcanti). Il riferimento alla vecchia bevitrice rinvia agli esempi latini di Oviclio e Properzio. Caratteristiche della poesia di Matteo sono la lasciviá e loscenità della vecchia, che possono trovare un antecedente in alcuni poeti toscani pre-burchielleschi come Francesco Scambrilla. ${ }^{17}$ Giovan Matteo di Meglio aggiunge agli insulti l'epiteto di ruffiana e prostituta, figura inedita nella tradizione comicá volgare, che troverà più ampio spazio rappresentativo nel Cinquecento. ${ }^{18}$ Matteo spesso usa l'aggettivo "maliosa" che secondo Brincat è sinonimo di strega. ${ }^{19}$ Nel sonetto "O chalandrona", la strega che prende parte alle tregende è definita "stregonizza", "maliosa" e "máestra di fatture." Spicca la lascivia della prostituta il cui appettito sessuale viene descritto con una crudezza riscontrata solo negli antecedenti latini, che possono avere ispirato Di Meglio. 20

Socchorso diogni frate e tregendiera,

vulua arrabbiata, in chui la foia è ntera,

anzi arsione, e di dricto e davante. (55)

Nel sonetto "S"tu volessi vectere un cloppio errore" (10-) "strega vecchica rinbanbita" è lappellativo attribuito alle donne che insistono nel volersi 
acconciare e truccare per parere più belle; così la tradizionale polemica misogina contro le donne che s'imbellettano si affianca qui al motivo della vecchiaia e della stregoneria. Matteo rinforza la sua ostilità contro la vecchia nella canzonetta "De! Udit'un po' novella"(51), dove la donna "grinza e vizza" fa da ruffiana alle proprie figlie. In questa ballatá il poeta denuncia pubblicamente i costumi depravati di una madre-mezzana e delle figlie prostitute, che hanno un amante diverso per ogni giorno della settimana. ${ }^{21}$ Di Meglio arrichisce il topos della vituperatio vetulae non solo aggiungendo la figura della strega, che era già nel Burchiello, ma soprattutto attribuendo alla vecchia gli attributi di prostituta/ruffiana lasciva.

Il vituperio della vecchia in area post-burchiellesca è attestato anche in Antonio Cammelli detto il Pistoia. Nonostante Cammelli trascorresse la maggior parte della sua vita fuori dalla Toscana, la sua vena creativa si situa nella poesia burlesca, nella caricatura, e nella poesia di impronta burchiellesca. Anche Pistoia utilizza il sonetto caudato, metro preferito dal Burchiello, e dimostra una particolare propensione per il grottesco: egli ama descrivere amici, nemici e se stesso in ritratti deformi. Cammelli dedica alle donne degli elogi convenzionali con qualche tono parodistico; famoso è il gruppo di sonetti (67-70) in cui loda le donne di varie città in cui visse (Firenze, Siena, Ferrara, Milano), ma affianca agli elogi anche delle caratteristiche difformi che contaminano la bellezza tradizionale. Quando la donna non è più giováne, essa diventa soggetto di più aperta ostilità e dell'invettiva. Il sonetto caudato "Il viene una imbrunata viduetta" inveisce contro una vedova di nobile casata che ha carni rancie, pelle crespa e faccia sudicia.

Il viene una imbrunata vicluetta, cliha quarantasette anni o manco un poco:

largo, brigata! orsù, dategli loco, tanto che "l passi via la sua carretta! Guardate occhietti come la civetta: che regina de scacchi posta al gioco! Lei pare un carboncin mezzo di foco: o che bel donnelin creato in fretta! Che belle carne purpurine e rancie! Quando le' aguzza quel bocchino strano fa mille crespettine ne le guancie. Lei par la fanticella di Vulcano, un giardinel clore nascon le ciancie; porta per galla un bocchettino in mano. Adesso parla piano, or si nasconde, or cenna, or ricle, or guarda. mostacin bel dá lavargliel in farda. 
Va via, che I foco te arda,

pulrida volpe ancor viva rimasa

per viluperio de sì nobil cassa! (193)

Di particolare interesse ie il dato anagralico (47 anni) che permette di apprendere con esaltezza l'età della donna vizza. Il tono del sonetto è un misto di invettiva e di lode paradossale ("che bel donnelin creato in fretta!") con alcuni elementi che accomunano questo testo alle parodie rusticane della Nencia da Barberino e della Beca da Dicomano, si pensi alluso dei diminutivi ("occhietti", "bocchino strano", "donnelino") caratteristico degli elogi comici della poesia rusticana. ${ }^{22}$ Qui però non si tratta, come nei testi nenciali, di donna amata, o di donna giovane, bensi di uná vecchia vedova dal viso crespo. Con tono derisorio l'autore invita la "brigata" a fare largo, non per ammirare la bellezzá della donna, ma perché essa passi in fretta ed esca di scena. Il sonetto si riallaccia alla tradizione vituperativa tramite l'invettiva finale, dove si augura alla vedova l'annientamento fisico. La morte al rogo e l'epiteto di "putrida volpe" (una delle tante trasformazioni della strega), permettono di inserire anche questa vecchia "viduetta" tra le streghe della poesia quattrocentesca. Mancano invece nel Cammelli lá componente oscena e la lascivia della vecchia prostituta caratteristiche del Di Meglio.

\section{Poliziano}

Oltre alla vasta cultura classica, nella sua opera poetica volgare Poliziano rivela un'estrema attenzione ed apertura sia verso la tradizione volgare aulica e comica, sia verso la letteratura toscana popolareggiante del suo secolo. Nella produzione volgare Poliziano rielabora i temi della tradizione popolare e soprattutto nelle ballate adotta un tono scherzosamente realistico. La ballata "Una vecchia mi vagheggia" riprende il topos della vecchia orrenda e lasciva, usando non tanto i toni aspri dell'invettiva quanto quelli comici della derisione. Questo testo, che si colloca nell'ambito del lusus, riunisce il motivo medievale della vecchia vizza e puzzolente a quello della prostituta, con riferimenti solo impliciti alla strega. Secondo Tateo questo testo riprende "il gusto della divertita rappresentazione della clonna disgustosa, che ritroviamo nella lirica latina." (183)

Una vecchia mi vagheggia,

vizza e secca insino all'osso;

non ha tanta carne adosso

che sfamassi una marmeggia.

Ell'ha logra la gingiva, 
tanto biascia fichi secchi, perch'e' fan della sciliva da 'mmollar bene e pennecchi: sempre in bocca n'ha parecchi, ché 'l palato se gli 'nvisca:

sempre al labro ha qualche lisca del filar ch'ella morseggia.

Ella sa propio di cuoio, quand'è concia, o di can morto, o di nidio d'avoltoio: sol col puzzo ingrassa l'orto (or pensate che conforto!) e fuggita è della fossa; sempre ha l'asima e la tossa e con essa mi vezzeggia.

Tuttavia el naso le gocciola, sa di bozzima e di sugna, più scrignuta è ch'una chiocciola: po', s'a un tratto el fiasco impugná, tutto 'l suga come spugna, e vuole anche ch'i la baci. Io la sgrido: "Oltre va' giaci!"; ella intorno pur matteggia. Non tien lanima coi denti, chun non ha per medicina; e luccianti ha quasi spenti, tutti orlati di tonnina. Sempre la virtù divina fin nel petto giù gli cola; rizza e secca è la suo golá, tal chun becco par d'acceggia. Tante grinze ha nelle gote, quante stelle sono in cielo; le suo poppe vizze e vote paion proprio ragnatelo. Nelle brache non ha pelo. della peccia fa grembiule; e più biasciál che le mule. quando intorno mi volteggia. (106-07)

Delcorno Branca individua con precisione gli antecendenti latini e volgari della ballata (191-92) a cui si devono aggiungere anche testi latini del Poliziano stesso, come il ritratto disgustoso di Febris dell'elegia "In Albieram" e la descriptio mulieris in negativo dellode "In Anum." Il lessico della ballata è intessuto di prestiti della tradizione realistica medievale: la vecchia è 
"Vizzal" come ba "vecchiuzza" di Nicola Muscia ed enette un "puzzo" cle rievoca la "buggeressa" di Rustico e la "recchiuzza" di Muscia; il "naso le gocciola", hal gli occhi "tutti orlati di tonnina" come la Beroe delle Artes, ed è "scrignuta" come la vecclaia del Cavalcanti. Il tono comico-grottesco di (questa ballata deriva dall'atteggiamento trasgressivo della vecchia orrenda colta nellatto di vagheggiare il poeta, "verho per eccellenza del galateo amoroso." 23 poliziano attribuisce alla vecchia alcune caratteristiche provenienti clalla tradizione popolare: la qualità di bevitrice e quella di filatrice, attività che per tradizione proverbiale viene attribuita alla prostituta che la lasciato il mestiere. Pur non definendo apertamente questa vecchia come stregà, sial l'attività di filatrice che l'attributo di bevitrice rinviano implicitamente alla prostituta e alla strega; nella realtà storica rinascimentale si attestano numerosi casi di prostitute hevitrici e assidue frequentatrici di taverne. Negli Amores ovidiani la vecchia prostituta Dipsa, oltre ad essere hevitrice insaziabile è anche a conoscenza di tutte le pratiche della stregoneria. ${ }^{2+}$ Questa ballata è una variante sullo schema tradizionale del vituperio descrittivo e dell'invettiva contro la vecchia ripugnante; come nel sonetto del Cammelli i) poeta colloquia con un uditorio che invita a partecipare alla derisione della vecchia. Secondo Delcorno-Branca dietro questa ballatá si profila la tradizione frottolistica contemporanea, in particolare la cosiddettá "Frottola degli innamorati" di Leon Battista Alberti, che riunisce molti elementi tematico-lessicali utilizzati nelle Rime del Poliziano. (195) La frottola albertiana include infatti una sfilata delle donne brutte, che può avere fornito a Poliziano una colorita galleria di ritratti femminili grotteschi.

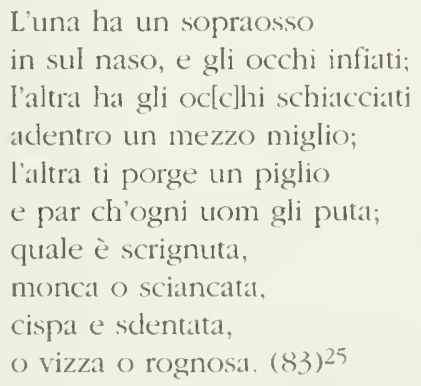

Le caratteristiche grottesche delle donne dell'Alberti rinviano agli antecendenti trecenteschi, basta ricordare ancora una volta la "scrignutuzza" di Cavalcanti e la diffusione dell’aggettivo "vizza" tra le recchie della poesia Medievale.

Poliziano sfruttá il motivo tradizionale del vituperio della vetula anche nel- 
lode latina "In Anum." Secondo i critici la ballata e l'ode latina rientrano nella categoria stilistica del lusus ma, mentre l'ode evidenzia "l'aspetto ributtante e libidinoso della vecchia, la ballata si muove esclusivamente sul piano del grottesco proverbiale e immaginoso" (Delcorno Branca, 194). Nell'ode latina il disgusto descrittivo, e la minuziosa attenzione alla bruttezza fisica della vecchia riprendono lo schema degli antecedenti letterari classici e della latinità medievale (Beroe).

Huc, huc, jambi! Arripite mi jam mordicus

Anum hanc furenti percitam libidine,

Tentiginosam, catulientem, spurcidam,

Gravedinosam, vietam, olentem, rancidam ${ }^{26}$

Come in Orazio la libidine è il motivo centrale dell'invettiva contro la vecchia prostituta. All'aspetto fisico repellente si aggiunge anche la qualità 'cadaverosa' della vecchia, definita in apertura "cadaverosam" e poi ancora "nec jam anus sed mortua." A questa vecchia turpe sono preferibili animali come la scrofa, l'asina ed il cane. ${ }^{27}$

\section{Conclusioni}

Questo articolo si collega al recente interesse della critica femminista per la vecchiaia femminile. Il pregiudizio di "gender" nei confronti della vecchiaia femminile è retaggio della tradizione classica e clella poesia volgare medievale: appare già nei vituperi latini di Orazio, Ovidio, Properzio e Marziale, e diventa vero e proprio topos letterario con la poesia comico-realistica di Rustico Filippi, pseudo-Angiolieri, Guido Guinizelli, Guido Cavalcanti, Nicola Muscia e Franco Sacchetti. Se la poesia giocosa due-trecentesca sfrutta il motivo clella vituperatio vetulae più come esercizio retorico che come espressione di un'effettiva avversione contro la vecchiaia femminile, i poeti toscani del XV secolo, arricchiscono il topos di motivi nuovi e rivelano l'ostilità caratteristica della temperie storico-culturale del loro tempo verso le vecchie che sono streghe e prostitute/ruffiane lascive. Nelle poesie di Burchiello, Matteo Di Meglio, Antonio Cammelli e Poliziano, l'immagine negativa della vecchiaia femminile non è più solo esercizio retorico su topoi letterari consolidati dalla tradizione, ma diventa espressione di un effettivo accanimento contro figure femminili altamente trasgressive. Le vecchie della poesia quattrocentesca sono attaccate con gli espedienti tradizionali dell'invettiva e della descriptio fisica repellente, ma sono anche rappresentate sempre più come lascive e impegnate in attività illecite: praticano arti magiche, sono prostitute e mezzane, sono il disonore clella famiglia, bevono all'ec- 
cesso, fanno avances erotiche al poeta. L'inveltiva si scaglia, con estrema violenza e con linguaggio talora crudo é volgare, contro queste vecchice orrende per il loro comportamento trasgressivo, che le rende oggetto non solo di derisione e vituperio ma anche di distruzione e annientamento corporeo.

\section{(Hile'sit)' of Alberta}

\section{NO'TE}

1 Il numero di Memoria è del 1986 e include l'articolo di "I seni di Eva. Immagini di vecchie nella pittura". In questo articolo Bianca Saletti concludeva che mentre gli uomini vecchi sono sempre rappresentati nella pittura come persone pience di dignità, le clonne sono spesso allegorizzazioni di cualità negative. Per una panoramica della tradizione letteraria sulla vecchia dai classici al Rinascimento si veda l'articolo di Bailló e anche il classico di Simone de Beauvoir, che però non si linnita ad esaminare solo la rappresentazione della reccliaia femminile.

2 Alla tradizione latina e comico-realistica Medievale è stato dato maggiore spazio in altra sede. Si veda il mio articolo "La vecchia guardiana e lo sguardo del poeta" di prossina pubblicazione negli atti del convegno dell'AATI di Crotone.

3 In area francese la vechia compare nei fabliaux (La Male Femme qui conchia la prude femme) e nel Roman de la Rose dove l'ipocrita Vielle finge di custoxtire la giovane e invece fa da mezzana. L'ostilità contro le vechie deriva anche dalla tradizione cavalleresca che nella poesia trovadorica celebra la bellezza giovanile e disprezza la vecchia. Per un esame più approfondito dei vari tipi di vecclia nella letteratura ronanza si veda Bailbé.

'I sonetti appaiono come anonimi nell'edizione di Sonetti burleschi e realistici nei primi due secoli a cura di Aldo Francesco Massera (310).

5 L'argomento assai vasto, che viene qui breventente riassunto, è il soggetto del primo capitolo del mio libro sulla donna brutta di prossina publolicazione presso l'editore Longo.

6 Le tre ballate (LXI- LXIII) riportate da Tommaso Casini nei suoi Studi di poesia anti$\mathrm{ca}$, si scagliano contro la vecchia guardiana dell'amata. In "La vechia d'anor m'a biasemata" alla guardiana molesta, maldicente e orrenda viene attribuito l'epiteto di strega c indovina e si augura di essere bruciata viva. La stessa sorte viene augurata alla guardiana della breve ballata "Do mala vechia, lo mal fuogo l'arda", mentre nella terza ballata "Laida vecchia stomegosa" e in una canzonetta adespota ("D'una vechia cliè zilosa") citata dal Cian (80-82), la vecchia è chiamata befana, una figura femminile che nella tradizione popolare era spesso associata alla strega. Anche la guardiana del madrigale "I na smaniosa e insensata vecchia", attribuito a Donato da Cascia (Corsi, 1047), viene chiamata "strega".

7 Ariosto si scaglia contro una vecelia prostituta lasciva nell'ode latina "In Lenam". Niccolò Franco compone it sonetto di invettiva contro "Una vecchiazza, ch'è tutta canuta", che con linguaggio osceno descrive te avances della vecchia al poeta. Il Molza compone "In anun importunam". Aretino poi, fa della prostituta il personaggio centrale clelle Sei giornate, dove la vechia prostituta consiglia alla giovane figlia di seguire la via della prostituzione.

8 In area veneta gli attacchi alla vecchia sono attestati già nella poesia di Leonardo Giustinian (1388-1+i6), che precede cronologicamente gli esempi toscani. Nelle varie 
tradizioni regionali esistono altri testi poetici dimettiva contro le vecchie. Si pensi ai due sonetti anonimi in dialetto nordorientale citati dial Cian e conservati alla biblioteca comunale di Ldine ("Veder ti possa rechia scarpelata", "Vedere ti possa rechia rabiosa") che saranno oggetto di studio in un successivo contributo. Ricordiamo anche la poesia cortigiana di Serafino Aquilano, che include una canzonetta contro una recchia, o la ballata anonima "Questa vecchia rimbambita" citata da Delcorno Branca (nota 116).

9 Ci limitiamo ad esaminare la figura della strega come compare nei testi poetici vituperativi. Streghe e stregoneria sono argomenti assai vasti e ben documentati che non è possibile trattare qui in forma esaustiva. Si rimanda al capitolo "L inquisizione e la donna" del libro di Romeo De Maio (Donna e Rinascimento. Milano: Il Saggiatore: 1987. 263-300) e alle nutritissime note. Richard Bernheimer ha messo in luce il legame tra l'aspetto fisico dellia strega e della donna selvatica. Secondo Bernheimer (35) nel vocabolario demonologico del Medio Evo strega e donna selvatica sono un'unica cosa. Nellantichità greca lamia è la donna orrenda che divora i bambini. nella Bibbia lamia appare col nome di Lilith. un mostro di sesso femminile; nel Medioevo molti autori fanno coincidere lamia con strix cioé la strega. Nel folklore medievale la donna selvatica ha un caratteristico aspetto fisico: è pelosa, talvolta di corporatura enorme, ha viso grinzoso e compare col corpo nudo ricoperto solo cli una folta capigliatura. In alcune rappresentazioni la mostruosità dell'aspetto fisico è contrassegnata dai seni penzolanti che si trascinano al suolo o che sono gettati dietro alla spalle (grinzosa coi seni penzolanti che toccano il suolo è descritta anche la vedova nel Corbaccio). Secondo Bernheimer (50-8t) nelle rappresentazioni teatrali medievali dei paesi alpini è frequente la caccia allorda selvaggia, che include una donna pelosa e selvatica. Le rappresentazioni dellorda selvaggia nel passato preistorico consistevano in un raduno di spiriti capeggiati da una divinita femminile che successivamente dà luogo al rituale del sabba delle streghe. Nel Medioevo la rappresentazione dellorda selvaggia faceva parte dei riti dell Epifania o del Carnevale. Epifania e Carnevale sono legati alla figura della recchia orrenda, una Befana che fa visita ai bambini clurante la dodicesima notte, e una strega bruciata l'ultimo giorno di Carnevale.

10 Nonostante in alcuni suoi sermoni professi l'ugugaglianza tra uomo e donna e sostengal i diritti femminili, Bernardino non transige sulla stregoneria. Per il suo accanimento contro le streghe si redano Origo (170-74), e Giovanni Battista Bronzini che esamina il tema della stregoneria nel sermone XXXV delle prediche senesi del 142-

11 Si veda l'articolo di Bonadiman che rintraccia l'origine storica della magia in area slava e trova documentazione risalente gia all'undicesimo secolo che conferma la particolare propensione delle donne a partecipare alla stregoneria.

12 Nella ballata LXI riportata clal Casini la vecchia è definita "striga" e "indorina", e nel madrigale "Una smaniosa e insensata recchia" di Donato da Cascia, presente nell'antologia di Rimatori del Trecento curata da Corsi, l'insulto è contro l' "arrabbiata strega". Nelle ballate anonime del XIV secolo pubblicate dal Casini la vecchia orrenda è barbuta come un uomo, e viene paragonata all'orso, uno dei travestimenti dellorda selvaggia. Nella canzonetta adespota "Lna rechia ch'è zilosa" riportata dal Cian la recchia pelosa e barbuta come un uomo è invece ura Befana.

13 Su questo punto concordano sia il Lanza (209) sia il Marti. Quest ultimo, in appendice a Cultura e stile nei porti giocosi del tempo di Dante, fa una rapida panoramica sugli sviluppi della poesia giocosa fino al Cinquecento. Burchiello è riconosciuto come lanello di congiunzione (ra Angiolieri e la poesia conica di Francesco Berni (21-).

1 s si notino le similarità con la descrizione disgustosa di Beroe "Aris sorde fluit, non orbiculata redundant Vermibus, huc illuc pendet obesa madens. Livescunt oculi, sanies decurrit, inundat, Fluxus, lippa regit lumina, faece replet . . Naris sima jacet, foetens, obliqua meatu. Distorto, flamen exitiale vomit" in Faral (131)

15 Questo sonetto dell'edizione di Londra (ma più probabilmente Lucca o Pisa del 1752) 
ci e perrenuto tramite il CD ckella $1.1 \%$ e non riportal alcuna numerazione della paginat. secondo la tradizione folkloristica la strega era associata ald alcuni animali tra cui andede il rospo o botta. si veda ad esempio Gittseppe Delfino, Aidano Schmuckher. 27.

16 In questo sonetto l'attenzione aghi oxchie al guardare rivelano che la vecclata e considerata dai poeti come un pericolo perché si sostituisce a loro nellatto di guardare. Questo ì il motivo centrate del mio articolo sulla vechia guardianal di prossima publalicazione.

$1^{-}$Nellantologial di Lirici toscani del Quatrocento, troviamo ad esempio it sonento di Francesco Scambrilla "Elle van col suggetlo alla spagnuola" (vol $11,4-3)$. Qui le denne vengono attaccate per la loro lascivial con toni crudi e linguaggio osceno. L'accumulazione di epiteti offensivi contro le donne, con la stessal sperimentazione dei suoni aspri presente in Giovan Matteo. include "vecchie ratorsate", "buggeresse" "maliose." Il finale fa riferimento alla presunta abitudine delle streghe di avere rapporti sessuali col cliavolo.

18 Numerosi sono i testi rinascimentali contro le prostitute d'alto loco o cortigiane, oltre alle già citate prostitute di Ariosto, Niccolò Franco e Aretino, ricordiamo l'inveltiva contro la meretrice Ortensia di Fancesco Coppetta Beccuti, e il capitolo "Contro una cortegiana" di Quinto Gherardo.

1) Si veda a pargina $5+$ la nota 2 di commento all edizione delle Rime clel di Meglio.

20 La connessione recchia-ruffiana-libidinosa è già presente nella commedia mediolatina. Si pensi allo pseudo-ovidiano De Vetula, dove una vecchia lasciva si offe di fare da mezzana ad Ovidio per il suo incontro amoroso con la giovane amata, ma poi con l'inganno è lei a sostituirsi all amata con la complicita del buio, facendo fuggire via Ovidio inorridito. Nel Pampbilus li mezzana è una recchia di brutto aspetto fisico. 11 motivo della mezzanat, vecchia ex-prostituta e maga trova la sua più alta espressione nella Celestina di Fernando Rojas, composta attorno al 1492.

21 Con Giovan Matteo il vituperio non si limita alle vecchie ma si estendle a qualunque donna brutta cli cui viene sottolineata la lascivia e la depravazione. I sonetti caudati "O chalandrona" e "O falsa ladra" insistono sullo smodato appetito sessuale della donnál vituperata senza indicarne la vecchiatia. In "O falsa ladra" la donna dell invettiva è chiamata "bagascia" e poi ancora "vacca c troia", immettendo una sfrontatezza e bassezza di linguaggio finora inedita. Il riferimento alla prostituta lasciva è presente in quattro su cinque dei componimenti comico-realistici di Giovan Matteo.

22 Nella seconda ottava della Beca, Pulci la descrive: "è solo un po piccina", "nellocchio ha in tutto una tal magliolina", "piloso ha intorno a quella suo bocchina" (II, 1, 3, 5).

23 Daniela Delcorno Branca giunge a definire questa vecchia un "ossimoro vivente"( 196), tanto contracklitoria è l'associazione della donna vecchia e orrenda al gesto di vagheggiare.

2. Si vedano i riferimenti proverhiali alla prostituta-filatrice citati da Delcomo Branca (200). Lyndal Roper, che conduce un indagine storico-sociale sulla prostituzione nel periodo rinascimentale, ha messo in eviclenza il particolare legame tra il bere e le prostitute. Poliziano ritorna sul tema della strega nella Lamia, la prolusione in prosia al corso sui Priora di Aristotele. Qui i maldicenti che si raggruppano a deridere lattivitit filosofical del Poliziano vengono assimilati ad un covegno di streghe; seblene ke streghe manchino della Caricaturaliti del testo poetico sono pur sempre descritte come filatrici.

25 Secondo Gorni il corteo grottesco delle donne fa parte, insieme ad altri personaggi della frottola, di "un grande atlante figurato di psicologia amorosa" ( 76 ).

26 Riportiamo a seguito il testo intero dell'ode. "Huc huc, jambi! Arripite ni jam mordicus Anum hane furenti percitam libicline, Tentiginosam, catulientem, spurcidam, Gravedinosam, vietam, olentem, rancidam, Cadaverosam, fronte rugosa, coma Cana atque rara, depilatis palpebris, Glabro supercilio, labellis defluentibus, Oculis rubentibus, genis lachrymantibus, Edentulamque (ni duo nigri et sordicli Dentes 
supersint),auriculis exanguibus/ Flaccisque, mucco naribus stillantibus, Rictu saliva undante, tetro anhelitu,/ Mammis senecta putridis praegrandibus/ Araneosis deciduis manibus,/ Laxoque ventre dissipato et fixili,/ Cunno ulceroso, verminante podice,/ Natibusque macris aridis et osseis,/ Utroque sicco crure utroque brachio,/ Talo genuque utroque procul extantibus,/ Calcaneoque pernionibus gravi;/ Ut nil sit aspernabilius, nil tetrius/ Monstrosiusque aut nauseabundum magis:/ Quam pistor olim, caupo, calo, bajutus, / Et institores, et lanius, et carnifex, / Et muliones permolebant et coci, / Ceu prostitutam et sellulariam meram;/ Nunc nemo jam vult visere nemo colloqui,/ Fastidit unusquisque et habet ludibrio/ Anum subante perditam prurigine:/ Sed audet impudens tamen, audet inmpudens,/ Procax, proterva, nec jam anus sed mortua,/ Utcumque prurit (prurit autem jugiter),/ Se postulare ut comprimam, sibi ut arrigam,/ Quasi ipse verres quasi asinus sim vel canis./ Abi hinc, abi, anus, in maximam malam crucem,/ Abi scelesta, obscoena; sive vera anus/ Seu terriculum es seu larva bustuaria./ Nam si optio mi detur, aedepol magis/ Scrofam futuan quam te vel asinam vel canem." (Opera Ommia. Torino: Bottega d'Erasmo, I970, 271-72)

27 Questo testo latino rivela una maggiore dipendenza dai precetti della retorica medievale e del ritratto di Beroe: il poeta si serve qui della descriptio nella sua forma conpleta, che passa in rassegna ogni parte del corpo della donna, rispettando il moto discendente dalla testa ai piedi. Il ritratto della vecchia presenta un 'canone lungo' delle bruttezze femminili, che enumera in dettaglio e con ricchezza d'aggettivazione l'intero corpo. Bettinzoli (179-80) ha rilevato le derivazioni di diretta provenienza oraziana (Epodo VIII 1-10). Questo ritratto ributtante defla vecchia sta in rapporto oppositivo con l'ode latina "In puellam", in cui Poliziano effettua, con forme altrettanto convenzionali, la commendatio pulchrae puellae. Il legame tra le due composizioni rinvia alla contrapposizione delle due figure femminili che emerge dalle Artes medierali e dalla tradizione comicoburlesca.

\section{OPERE CITATE}

Alberti, Leon Battista. Rime e zersioni poetiche. A cura di Guglielmo Gorni, MilanoNapoli: Ricciardi, 1975.

Bailbé, Jacques. "Le Thème de la vieille femme dans la poésie satirique du seiziéme et du début du dix-septiéme siécle" Bibliotheque d'Humanisme et Renaissance 26 (1964): 98-119.

de Beauvoir, Simone. Old Age. London: Penguin, 1977.

Bernheimer, Richard. Wild Men in the Middle Ages: A Study in Art, Sentiment, and Demonology. Cambridge: Harvard UP, 1952.

Bettinzoli, Attilio. "Dolus et Error': di alcuni carmi latini del giovane Poliziano" Lettere italiane 382 (1986): 166-92.

IBonadiman, Beatrice. "Ogni vecchia è una strega'. Origine storica della magia in area slava" Geografia storia e poetiche det fantastico. Firenze: Olschki, 1995, 61-71.

Bronzini, Giovanni Battista. "La predicazione di Bernardino da Siena fra scrittura e oralità" San Bernardino da Siena predicatore e pellegrino. Atti del comegno nazionale di studi bernardiniani. Maiori, 20-22 ginngo 1980). Galatina: Congedo, 1985, 129-50.

Burchiello [Domenico di Giovanni]. Sonetti editi e inediti. A cura di Michele Messina. Firenze: Olschki, 1952.

Cámmelli, Antonio. I sometti faceti di Antonio Cammelli. Secondo l'antografo ambrosiano. A cura di Erasmo Pèrcopo, Napoli: Jovene, 1908. 
Casini, Tommaso. Studi di presia antica. Città di Cáslello: Lápi, 1913.

Cian Vittorio, "Un codice ignoto di rime volgari appartenuto at B. Cástiglione" GSLI 3.t (1899): 297-353.

Corsi, Giuseppe. Rimatori del Trecento. Torino: L.T.T.T, 1969.

Delcomo Branca, Daniela. "Il laboratorio del Poliziano. Per una lettura delle Rime." Lettere Italiane 39 2 (1987): 190-201.

Delfino, Giuseppe, Schmuckher, Aidano. Stregoneria, magia, credenze esuperstizioni a Genoua e in Liguria, Firenze: Olschki, 1973.

Faral, Edmond. Les Arts l'oćtiques du XIIt' et du XIIle Siecle. Recherches et Documents sur la Téchnique Littéraire du Moyen Age. Paris: Champion, 1982.

Feminist Encyclopedia of Italian Literature. "Witch", di Laturen Lee. Westport CT: Greenwood Press, 1997, 355-57.

Frugoni, Chiara. "The imagined Woman" A History of Women in the West. II Silences of the Niddle Ages. Cambridge, London: Belknap of Harvard LP, 1992, 336-422.

Lanza, Antonio. Polemiche e berte letterarie nella Firenze del primo Quattrocento. Roma: Bulzoni, 1971.

- "Aspetti e figure della poesia comico-realistica toscana del secolo XV" La Rassegna della Letteratura Italiana 898 (1985): 403-43.

Lirici toscani del Quattrocento. A cura di Antonio Lanza. Roma: Bulzoni, vol. I 1973 , vol If 1975.

LIZ, Letteratura italiana Zamicbelli. CD. Bologna: Zanichelli,1993.

Marotti, Ornella. "Age and Ageism: Facts and Fiction" Italian Culture 15 1997: 193204.

Marti, Mario. Cultura e stile nei poeti giocosi del tempo di Dante. Pisa: Nistri-Lischi, 1953.

Massera, Aldo Francesco. Sonetti burleschi e realistici dei primi due secoli. Bari: Laterza, 1940.

di Meglio, Giovan Matteo. Rime. A cura di Giuseppe Brincat. Firenze: Olschki, 1977.

Origo, Iris. The World of San Bernardino. New York: Harcourt Brace, 1962.

Poliziano, Angelo. [Angelo Ambroginil. Rime. A cura di Daniela Delcorno Branca. Venezia: Marsilio, 1990.

Rimatori del Trecento. A cura di Giuseppe Corsi. Torino: U.T.E.T, 1969.

Roper, Lyndal. "Madri di depravazione. Le mezzane nel Cinquecento." Memoria 17 (1986): 7-23.

Tateo, Francesco. "Angelo Poliziano" Il Quattrocento. L'età dell'umanesimo. Bari: Laterza, 1972, vol. III, tomo 2, 155-245. 